\title{
Erratum a: La sindrome da carcinoide
}

\section{Roberto Baldelli ${ }^{1}$. Valerio Renzelli ${ }^{2}$. Francesca Rota ${ }^{1}$ • Paola Di Giacinto ${ }^{1}$. Antonio Bianchi ${ }^{3}$. Laura Rizza ${ }^{1}$. Paolo Zuppi ${ }^{1}$}

\section{Erratum to: L'Endocrinologo} https://doi.org/10.1007/s40619-020-00653-y

La versione originale di questo articolo purtroppo conteneva un errore. I nomi di due farmaci sono stati erroneamente inseriti nella tabella al momento della pubblicazione iniziale.

La tabella corretta è riportata di seguito e la tabella dell'articolo originale è stata aggiornata.

L'editore desidera scusarsi per questo errore.
Nota della casa editrice Springer Nature rimane neutrale in riguardo alle rivendicazioni giurisdizionali nelle mappe pubblicate e nelle affiliazioni istituzionali.

QUADRO SINOTTICO DEI FARMACI CITATI

\begin{tabular}{lll}
\hline Principio attivo & Nomi commerciali & Dosaggi \\
\hline Octreotide & Sandostatina & fl $50 \mathrm{mcg} / \mathrm{ml}, 100 \mathrm{mcg} / \mathrm{ml}, 500 \mathrm{mcg} / \mathrm{ml} ; \mathrm{sc}$, ev \\
Octreotide LAR & Sandostatina LAR & $1 \mathrm{fl} 10 \mathrm{mg}, 20 \mathrm{mg}, 30 \mathrm{mg} ; \mathrm{im}(\mathrm{ogni} 28 \mathrm{giorni})$ \\
Lanreotide & Ipstyl & $1 \mathrm{fl} 60 \mathrm{mg}, 90 \mathrm{mg}, 120 \mathrm{mg} ; \mathrm{im}(\mathrm{ogni} 14,21,28 \mathrm{giorni})$ \\
Telotristat & Xermelo & cp $250 \mathrm{mg} ;$ os \\
Pasireotide & Signifor & fl $0,3 \mathrm{mg}, 0,6 \mathrm{mg}, 0,9 \mathrm{mg} ; \mathrm{sc}$ \\
Everolimus & Afinitor & cp $2,5 \mathrm{mg}, 5 \mathrm{mg}, 10 \mathrm{mg}:$ os \\
${ }^{177}$ Lu-DOTATATE & Lutathera & fl $370 \mathrm{MBq} / \mathrm{mL} ;$ ev \\
${ }^{131} \mathrm{I}-\mathrm{MIBG}$ & Iobenguano ${ }^{131} \mathrm{I}$ & fl $185 \mathrm{MBq} / \mathrm{ml}, 246,7 \mathrm{MBq} / \mathrm{ml}, 493,3 \mathrm{MBq} / \mathrm{ml} ;$ ev \\
\hline
\end{tabular}

Per l'elenco completo dei farmaci citati e le relative schede tecniche si rimanda al prontuario dei farmaci. $o s$, orale; $s c$, sottocutaneo; $e v$, endovena; $i m$, intramuscolo; $c p$, compresse; $f$, fiale

The original article can be found online at https://doi.org/10.1007/ s40619-020-00653-y

$凶$ R. Baldelli

r.baldelli@scf.gov.it; roberto.baldelli@uniroma1.it

1 Unità di Endocrinologia, A.O. San Camillo-Forlanini, Roma, Italia

2 Unità di Endocrinologia, Dipartimento di Medicina Sperimentale, "Sapienza" Università di Roma, A.O. S. Andrea, Roma, Italia

3 Unità di Endocrinologia, Università Cattolica "Sacro Cuore", Policlinico A. Gemelli, Roma, Italia 